\title{
Modeling and simulation performance analysis of wind tunnel electric putter control system
}

\author{
Zhida Zhu ${ }^{*}$, Li Zeng, Haijiang Kou, Fan Zhang, Min Dai, Jian Yang, Wenjun Wang \\ School of Mechanical Engineering, Yangzhou University, Yangzhou and 225127, China \\ *Corresponding Author: zdzhu@yzu.edu.cn
}

\begin{abstract}
In a hypersonic wind tunnel, the motion of the electric putter Y mechanism needs to be controlled synchronously, and the accuracy or control performance of the system's synchronous motion control directly affects the running quality of the wind tunnel. Based on the deviation coupling mechanism of output displacement, the paper puts forward the synchronous control system of the model mechanism electric putter Y mechanism, and establishes the dynamic model of the mechanical system, current control ring, speed control ring and position control ring of the electric putter $Y$ mechanism. A closed-loop synchronous three-ring control system is designed, and performance simulation is carried out. The results show that the system has fast synchronous response speed, high accuracy of synchronous displacement and synchronous speed control, and the system has strong robustness.
\end{abstract}

Keywords: wind tunnel; model mechanism; synchronous control; mathematical model; performance analysis

\section{Introduction}

Wind tunnel is a test device used to simulate the movement of aircraft in the atmosphere, for the model to carry out force test, heat test, wind tunnel field test test, stage separation and multibody separation test. The wind tunnel device has a synchronous control system that supports the synchronous motion of the support mechanism, which has a control accuracy or control performance, which directly affects the operation quality of the wind tunnel. At present, the synchronous control system mainly has the control strategies such as parallel synchronous control, main command reference synchronous control $^{(1)}$, main-machine synchronous control ${ }^{(2)}$, and cross-coupling synchronous control, wherein the coupling synchronous control strategy includes the cross-coupled type ${ }^{(3)}$, the adjacent cross-coupled control ${ }^{(4)}$, the deviation coupling ${ }^{(5)}$, and other forms of coupling different control strategies. Parallel control is one of the simplest and direct synchronization control strategies. Its advantage is that the synchronization performance of the system is better in the start-up and stop phase, but the whole system is open-loop control. When one of the subsystems is disturbed in the running process, There will be a great deviation between subsystems, that is, the synchronization performance of the whole system is poor. The master-slave synchronization control strategy takes the output of the master subsystem as the input of the slave subsystem, and any reference input or disturbance loaded on the master subsystem will be reflected and followed by the slave subsystem. However, any disturbance from the subsystem will not be fed back to the master subsystem, so when the load of the slave subsystem changes, the synchronization accuracy between the subsystems can not be guaranteed. The cross-coupling synchronization control strategy is mainly to the output of the subsystems of the velocity or displacement, comparing two synchronization error, and attach it as a reference input signal feedback to each subsystem of control circuit, and its compensation, to reflect any subsystem of load changes, thus make the system get good synchronization control precision. However, the synchronous control strategy for wind tunnel model mechanism is mainly parallel synchronization control strategy. The control method is simple and low cost, and is mainly used in the case of high stiffness of synchronous connection mechanism. In this paper, based on the synchronization accuracy requirement of the electric push rod Y synchronous mechanism system in a wind tunnel, the mathematical model of the mechanism synchronization control system is established by using the cross coupling synchronization control strategy, and the simulation performance of the system is analyzed. In order to realize the synchronous motion of $\mathrm{Y}$ mechanism with high precision. 


\section{Mathematical model of wind tunnel}

\section{electric putter control system}

\subsection{Composition of the wind tunnel electric putter control system}

The structure of the electric putter $\mathrm{Y}$ mechanism control system of a wind tunnel model is shown in Figure 1 as a two-axis synchronous control system. The system is a cross-coupled synchronous control system consisting of the active axis PID closed-loop control system and the driven axis PID closed-loop control system. The closed-loop control system consists of PID control regulator, motor driver, servo motor system and its current ring control system, speed ring control system and its detection system, driven Y mechanism and its displacement detection system.

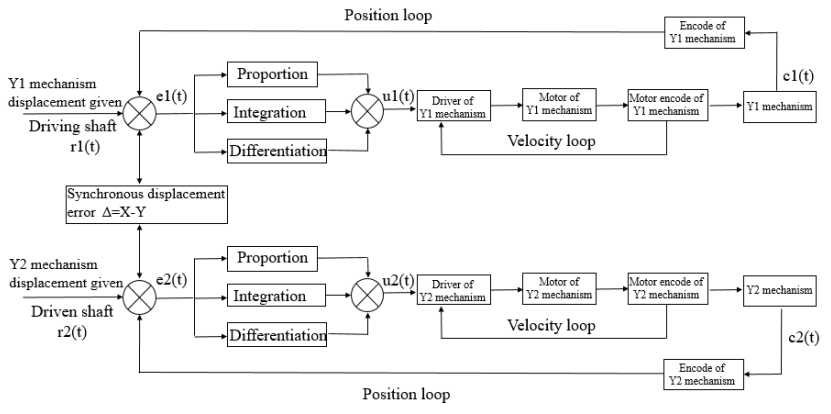

Fig. 1. Y mechanism synchronous control system

The electric putter $Y$ mechanism of the wind tunnel model is shown in Figure 2. Its mathematical models include the load mechanisms such as pushrod-driven load motion, screw nut power drive, screw rotation, and mathematical relationships or equations for permanent magnetic synchronous motor (PMSM) power drive units.

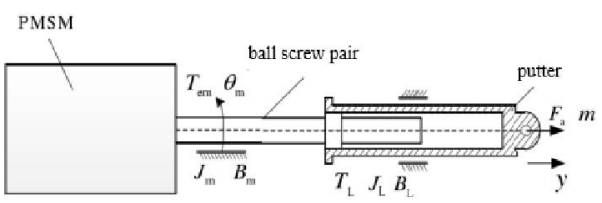

Fig. 2. Electric putter mechanism diagram

\subsection{Dynamic equations for load mechanisms}

The nut, pushrod and other load power parameters such as the pushrod mechanism drive load along the $\mathrm{Y}$ direction to make straight motion, and the Y-oriented end moving mechanism of its drive, are converted to the motor output shaft by the ball screw drive, and the equivalent integrated load torque $T_{L}$ and equivalent integrated load rotation $J_{L}$ inertia of the electric putter Y mechanism of the wind tunnel model are respectively.

$$
\left\{\begin{array}{l}
T_{L}=\frac{F_{a} \cdot P_{h}}{2 \pi \eta} \\
J_{L}=J_{s}+m \cdot\left(\frac{P_{h}}{2 \pi}\right)^{2}
\end{array}\right.
$$

In the form, $P_{h}$ is the vice-lead of the ball screw nut, $\eta$ is the positive efficiency of the ball screw nut sub-feed, $F_{a}$ is the power of the pushrod to drive the load mechanism, $J_{s}$ is the rotation inertia of the ball screw nut sub-bar shaft, and $m$ is the total mass of the moving assembly and the drive load. At the same time, the relationship between the rotation angle shift $\theta_{m}$ of the motor-driven ball screw shaft and the straight displacement of the nut pushrod $y$ is

$$
\theta_{m}=\frac{2 \pi}{P_{h}} y
$$

The inertia of the system and the viscous damping coefficient are respectively

$$
\left\{\begin{array}{l}
J_{e}=J_{m}+J_{L} \\
B_{e}=B_{m}+B_{L}
\end{array}\right.
$$

In the formula, $J_{e}$ and $B_{e}$ are converted to the equivalent inertia and equivalent sticky coefficient to the end of the motor shaft, and $J_{m}$ and $B_{m}$ are the inertia and viscous coefficient of the motor rotor, respectively.

According to Newton's second law wind tunnel model electric putter mechanical system dynamics equation is

$$
\left\{\begin{array}{l}
T_{e m}-T_{L}-B_{e} \dot{\theta}_{m}=J_{e} \ddot{\theta}_{m} \\
y=\frac{P_{h}}{2 \pi} \theta_{m}
\end{array}\right.
$$

In the formula, $T_{e m}$ is the electromagnetic torque generated by the motor; and $\dot{\theta}_{m}=\omega_{r}$ is the mechanical angular velocity of the motor rotor and its connected screw shaft, which is present with the motor's electric angle speed $\omega: \omega=p_{n} \omega_{r}$

\subsection{Dynamic equations of permanent magnetic synchronous motors}

The parameters of the permanent magnetic synchronous motor used by the wind tunnel model 
mechanism are shown in Table 1.

Table 1 Permanent Magnetic Synchronous Motor Parameters

\begin{tabular}{ccc}
\hline Parameters & Values \\
\hline Operating Voltage & $U_{d c} / \mathrm{V}$ & 56 \\
\hline Rated current & $/ \mathrm{A}$ & 20.4 \\
\hline Rated torque $\quad T_{e m} / \mathrm{N} \cdot \mathrm{m}$ & 2.8 \\
\hline Rated speed $\quad n / \mathrm{rpm}$ & 3000 \\
\hline Rated power $\quad P_{N} / \mathrm{W}$ & 880 \\
\hline Number of pole pairs & $p_{n}$ & 5 \\
\hline Phase resistance of the stator winding & $R_{m} / \Omega$ & 0.085 \\
\hline Quadrature axis inductor & $L_{q} / \mathrm{mH}$ & 0.121 \\
\hline Direct axis inductor & $L_{d} / \mathrm{mH}$ & 0.123 \\
\hline Permanent magnetic chain & $\psi_{f} / \mathrm{Wb}$ & 0.014224 \\
\hline Equivalent viscosity coefficient & $B_{e} / \mathrm{N} \cdot \mathrm{m} /(\mathrm{rad} / \mathrm{s})$ & $7.44 \times 10^{-4}$ \\
\hline Equivalent moment of inertia & $J_{e} / \mathrm{kg} \cdot \mathrm{m}^{2}$ & $1.38 \times 10^{-3}$ \\
\hline
\end{tabular}

Figure 1 shows the synchronous control system of the electric pushrod Y mechanism, the power drive device is the AC servo permanent magnetic synchronous motor (PMSM) system. In order to simplify the system, the necessary hypothesis simplification is made when the mathematical model of the permanent magnetic synchronous motor is established, and the control method of d-axis component current $i_{d}=0$ is used, the differential equation of the motor can be expressed as a single input-single output motor system, i.e

$$
\left\{\begin{array}{l}
u_{q}=R_{m} i_{q}+L_{q} \frac{d i_{q}}{d t}+p_{n} \omega_{r} \psi_{f} \\
T_{e m}=\frac{3}{2} p_{n} \psi_{f} i_{q}=k_{t} i_{q}
\end{array}\right.
$$

In the formula, $u_{q}, i_{q}$, and $\psi_{f}$, respectively, the stator $q$-axis voltage, current, and the components on the permanent magnetic chain $q$-axis; $L_{q}$ is $q$-axis inductor, $p_{n}$ is motor pole-positive, $k_{t}$ is electromagnetic torque constant.

\subsection{Control model of electric pushrod drive $\mathrm{Y}$ mechanism}

The system shown in Figure 1 is a two-axis synchronous control system, and the structure of each axis system is identical, so only one of the axis systems needs to be used to establish its control structure model. Take the Laplace transform of equations (4) and (5) to draw the open-loop control system of the electric push rod control system, as shown in figure 3 .

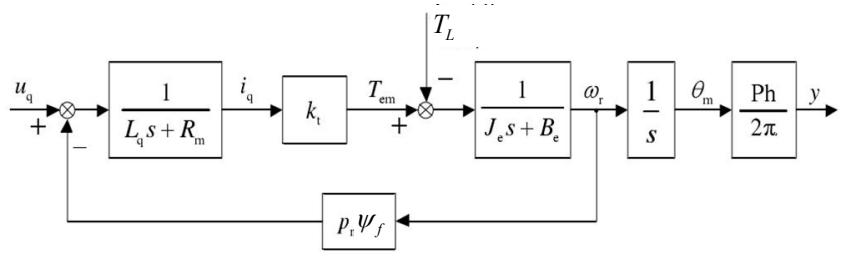

Fig. 3. Structure of the electric pushrod open-loop control system

According to the requirements of the synchronous control of the electric putter Y mechanism, the system is a typical position servo system, based on the detected Y-to-position signal to control the precise tracking of the position of the system. The system is a closed-loop control system controlled by three rings, such as current ring, speed ring and position ring. The current ring is used to transform the transfer function of the inner ring control object to improve the system's fastness, quickly suppress the internal and anti-potential interference of the current ring, and limit its maximum current, so that the system has sufficient acceleration torque, and ensure the safe operation of the system; The speed ring is used to suppress the influence of load disturbance, inertia change and pushrod thrust fluctuation, improve the speed control accuracy of the system and improve the dynamic response performance of the whole system; As the outermost position ring of the system is the most important link in the system, the accurate tracking of the output position of the system putter is realized through position detection, and the target is controlled precisely. Think of the inverter that drives the motor as an inertial link, i.e.

$$
G_{p w m}(s)=\frac{K_{p w m}}{T_{p w m} s+1}
$$

In the formula, $T_{p w m}$ is the time constant of the inverter power supply, an $K_{p w m}$ is the inverter magnification factor.

The establishment of an electric pushrod single-axis control system for three-ring control, such as current, speed and position, is shown in Figure 4. In the figure, take the inverter amplification factor $K_{\mathrm{pwm}}=1$, current feedback amplification coefficient $\gamma=1$, speed feedback amplification coefficient $\beta=1$, position feedback amplification coefficient $H=1$, current ring time constant $T_{\mathrm{oi}}=62.5 \mu \mathrm{s}$, inverter time 
constant $T_{\text {pwm }}=62.5 \mu \mathrm{s}$. Both the current ring and the velocity ring are regulated by the PI regulator ACR, which transmits the function.

$$
G_{c}(s)=\frac{K_{p}\left(T_{i} s+1\right)}{T_{i} s}
$$

In the formula, $K_{p}$ is the proportional adjustment parameter of the current ring or speed ring, and $T_{i}$ is the integral time adjustment parameter of the current ring or speed ring.

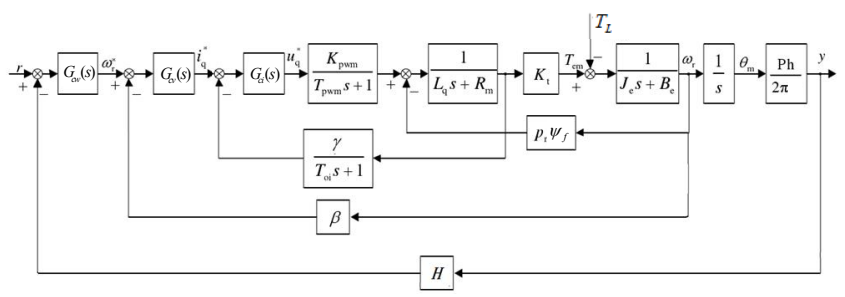

Fig. 4. Single-axis three-ring control system

The position ring regulator of the electric pushrod single-axis control system is used with PID regulator, which has a transfer function of

$$
G_{c}(s)=\frac{K_{w}}{T_{w i}} \cdot \frac{T_{w i} T_{w d} s^{2}+T_{w i} s+1}{s}
$$

In the formula, $K_{w}$ is the proportional adjustment parameter, $T_{w i}$ is the integral time adjustment parameter, and $T_{w d}$ is the differential time adjustment parameter.

\section{Simulation performance analysis of}

\section{the synchronous control system of}

\section{electric putter}

\subsection{Simulation performance analysis of current ring control}

The performance of the electric putter control system of the wind tunnel model depends on the vector control strategy of the permanent magnetic synchronous motor. In the final analysis, the control of the motor stator current, high-performance electromechanical servo system performance depends on the optimal performance of each ring, and the performance of the outer ring depends on whether the inner ring performance is optimal. Therefore, the current ring occupies an important position in the whole electromechanical position servo system, which is the key to improve its response speed, control accuracy, and improve the performance of the system. The permanent magnetic synchronous servo motor uses the voltage inverter as the control object of the current ring, and its transfer function is shown in the equation (6). Because its current feedback signal contains a large number of harmonic components affecting the stability of the system, the harmonic component is filtered by adding inertia (filtering) in current feedback, and the inertia link of the time constant equal is set at the given input of the current ring to compensate for the time delay caused by the filtering link, and its transfer function is

$$
G_{0 i}(s)=\frac{1}{T_{o i} s+1}
$$

If the control system meets the conditions: 1) the current ring response speed is fast enough, the cross feedback item $p_{n} \psi_{f}$ in Figure 5 can be ignoreda; 2) the equivalent stator winding electromagnetic time constant $T_{\mathrm{m}}$ is much larger than the power switch tube dead zone time and switch delay time $T_{\text {pwm }}$ and the current ring feedback channel filter time constant $T_{\mathrm{oi}}$. The open-loop transfer function of the current ring is

$$
G_{i k}(s)=\frac{K_{i}\left(T_{i} s+1\right)}{R_{m} T_{i} s\left(T_{p i} s+1\right)\left(T_{m} s+1\right)}
$$

In the formula, $T_{m}=L_{q} / R_{m}$ is the electromagnetic time constant of the synchronous motor; $T_{p i}=T_{p w m}+T_{o i}$.

Eliminate the maximum time constant with a PI regulator to improve the response speed of the current ring. Select the integral time constant $T_{\mathrm{oi}}=T_{\mathrm{m}}=0.00142 \mathrm{~s}$ of the PI regulator, correct the current ring to type I second-order system, by the formula (10) closed loop transfer function is

$$
\Phi_{i}(s)=\frac{K_{I i}}{T_{p i} s^{2}+s+K_{I i}}
$$

In the formula, $K_{I i}=K_{i} /\left(T_{i} R_{m}\right)=K_{i} / L_{q}$ is the open-loop pass gain of the current ring.

The closed-loop system of the current ring is compared with the second-order system in the standard form, and the system is parameterised with the second-order optimal law (the optimal damping ratio is 0.707). That is, the optimal damping ratio of the current ring system is 0.707 , then the formula (11) is available

$$
\omega_{n}^{2}=\frac{K_{I i}}{T_{p i}}, 2 \zeta \omega_{n}=\frac{1}{T_{p i}}
$$


The formula (12) is solved: . Thus, the control parameters of the available PI regulator are: integral time adjustment coefficient $K_{i}=L_{q} K_{I i}=0.121$; Scale adjustment factor $K_{i}=L_{q} K_{I i}=0.121$; The performance of the obtained current ring based on MATABL simulation is shown in Figure 5 by replacing each parameter (12). The unit step response curve shown in Figure 5(a) shows that the current rise time is about $0.6 \mathrm{~ms}$, the maximum overshoot is about $4.6 \%$, so the system meets the dynamic requirements. The open-loop Bode diagram shown in Figure 5(b) shows that the gain margin of the system is infinity, the phase margin is $65.5^{\circ}$, and the amplitude crossing frequency is $3.64 \times 10^{3}$ $\mathrm{rad} / \mathrm{s}$, and the visible current ring has better dynamic and steady-state performance.

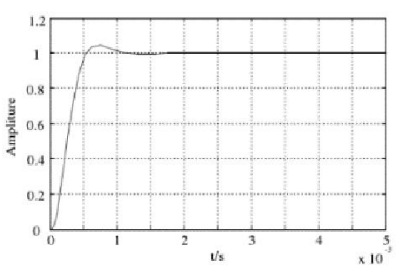

(a) Step response curve

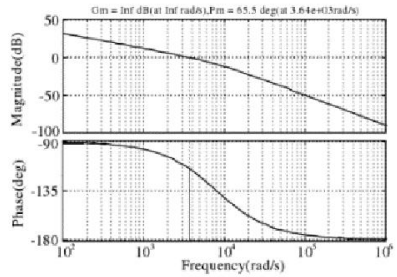

(b) Bode diagram
Fig. 5. Performance graph of the current ring

\subsection{Simulation performance analysis of speed ring control}

The regulator of the velocity ring is the current ring, which transforms the current ring closed-loop transfer function (11) into

$$
\Phi_{i}(s)=\frac{1}{2 T_{p i}^{2} s^{2}+2 T_{p i} s+1}
$$

In the upper formula, $T_{p i}=1 / 2 K_{I i}$. For speed regulator parameter optimization and performance analysis, the current ring of the control adjustment object can be lowered. When the open-loop cut-off (amplitude crossing) frequency $\omega_{c v} \leq 1 /\left(5 T_{p i}\right)$ of the speed ring, the transfer function of the current ring can be approximated to the first-order inertia link of the time constant $\tau_{i}=2 T_{p i}$, i.e. $\mathrm{g}$ $\Phi_{i}(s) \approx \frac{1}{2 T_{p i} s+1}=\frac{1}{\tau_{i} s+1}$. Thus, the open-loop transfer function of the speed ring before adjustment is

$$
G_{k 0 v}(s)=\frac{K_{t} / B_{e}}{\left(\tau_{i} s+1\right)\left(T_{e} s+1\right)}
$$

In the formula, $T_{e}=J_{e} / B_{e}$ is the electromechanical time constant of the speed ring.

In the absence of a regulator, the speed ring is unstable and needs to be comprehensively corrected, with PI regulator (7) to turn the speed ring into a type I system, with the open loop transfer function is

$$
G_{k v}(s)=\frac{K_{I v}\left(T_{v} s+1\right)}{s\left(\tau_{i} s+1\right)\left(T_{e} s+1\right)}
$$

In the formula, $K_{I v}=K_{t} K_{v} /\left(B_{e} T_{v}\right)$ is the open-loop gain of the speed ring.

By phase margin $\gamma>30^{\circ}$, the speed ring regulator parameters $K_{I v} \leq 10, T_{v} \leq 2 \times 10^{-4}, K_{v} \leq 8.943 \times 10^{-6}$ are simulated, and the speed ring performance curve is shown in Figure 6. As can be seen from Figure 6(a), the phase margin of the system $\gamma=31^{\circ}$, the cut-off frequency is $\omega_{c}=2810 \mathrm{rad} / \mathrm{s}$, the gain margin is infinity, and figure 6(b) can be seen that the system rise time of its unit step response is about 0.6 milliseconds, indicating that the system's dynamic response speed, stability is good and the steady state error is zero, but the overshoot amount is $40 \%$. This can be solved with a PID regulator in the position control system of the outer ring.

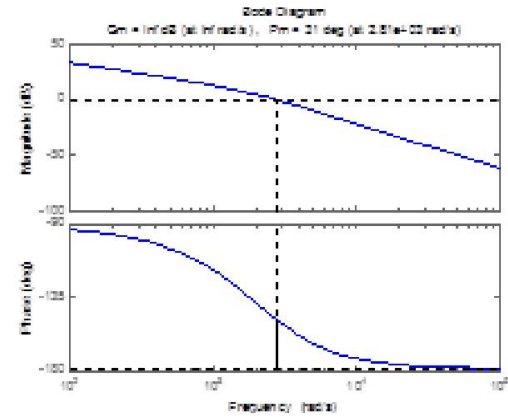

(a) Open-loop-to-peer frequency characteristic curve of the speed ring

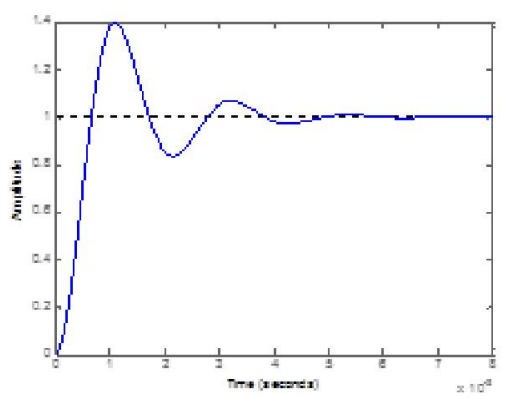

(b) Closed-loop unit step response curve for speed ring

Fig. 6. Performance graph of the speed ring

3.3 Simulation performance analysis of the synchronous control system of electric putter 
The outer ring of the wind tunnel electric pushrod synchronous control system is the position ring. The control object sits on the position ring, which includes the speed ring and the end of the pushrod ited, the Y-toward mechanism, which is adjusted by PID to form a 5th-order TYPE II system, whose open-loop transfer function is

$$
G_{k w}(s)=\frac{K_{I w}\left(T_{v} s+1\right)\left(T_{w i} T_{w d} s^{2}+T_{w i} s+1\right)}{s^{2}\left(\tau_{i} T_{e} s^{3}+\left(\tau_{i}+T_{e}\right) s^{2}+\left(1+K_{I v} T_{v}\right) s+K_{I v}\right)}
$$

In the formula, $K_{I w}=\frac{K_{w} P_{h} K_{I v}}{2 \pi T_{w i}}$ is the open-loop gain of the system.

The 2 PID regulators of the position ring control system are parameter-adjusted, and the proportional adjustment parameter $K_{I w}=150$, the differential time adjustment parameter $T_{w d}=1.4999 \times 10^{-4}$, and the integral time adjustment parameter $T_{w i}=6.2495 \times 10^{-4}$ are obtained.

(1) Simulation performance analysis of the displacement synchronous control of the electric putter system

Based on the control model, Figure 1 and Figure 4 of the aforementioned electric putter system, the simulation model of the double electric putter closed-loop coupling contract step control system is established, and the performance curve of the electric putter $\mathrm{Y}$ mechanism synchronous control system can be obtained by simulating the system as shown in Figure 7.
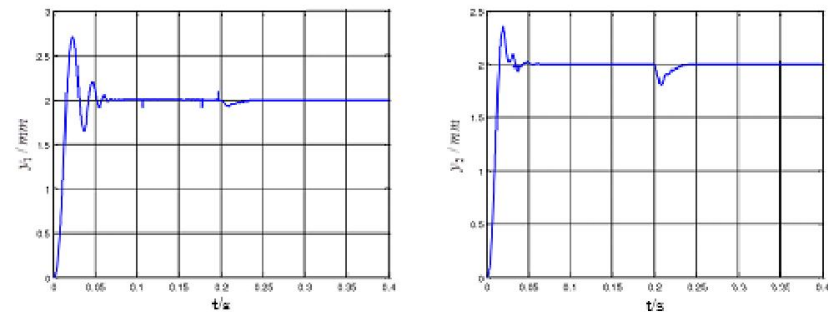

(a) Y1-to-displacement step response (b) Y2-to-displacement step response

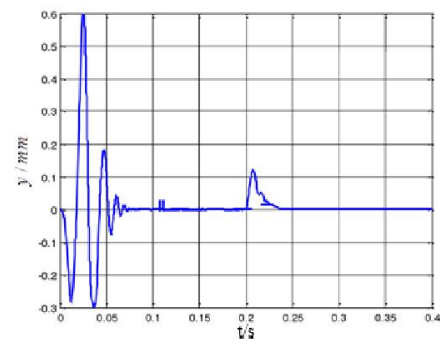

(c) The main, driven axis displacement

Fig. 7. Displacement response characteristic curve for electric putter synchronization system
Figure 7 is a step response graph for the output of the driven axis Y2, which is suddenly disturbed by the load. Figure 7(a) is the step response performance graph for the active axis, and Figure 7(b) is the step response performance graph for the driven axis. Comparing 2 graphs shows that the synchronization error of the system is compensated and has good dynamic response performance. Figure 7(c) is the synchronous error response curve of the system used for disturbance action, the maximum dynamic error is not more than $0.6 \mathrm{~mm}$, indicating that the dynamic error of the system is small, less than $1.2 \mathrm{~mm}$ required by the system, and the steady-state error is zero, and has good anti-jamming ability.

(2) Simulation performance analysis of speed synchronous control of electric putter system

When the wind tunnel model body electric putter system is given a reference speed of $300 \mathrm{~mm} / \mathrm{s}$, when the system reaches a steady state, that is, when the simulation time is $0.15 \mathrm{~s}$, the active axis $\mathrm{Y} 1$ suddenly applies a interference load of $50 \mathrm{~N}$ size, the step response curve of the active axis Y1 and the derived axis Y2 output speed and its synchronous control error response curve are shown in Figure 8 (a). From the figure, the maximum driving speed ( $M_{p}=16.7 \%$ ) of the active axis Y1 mechanism is smaller than the maximum driving speed $\left(M_{p}=36.7 \%\right)$ of the driven axis Y2 mechanism, but after $0.075 \mathrm{~s}$ both reach a steady state, and its speed is steady." All are $300 \mathrm{~mm} / \mathrm{s}$, and after applying a interference load on $\mathrm{Y} 1$ at $0.15 \mathrm{~s}$, the speed of the main spin-off axis fluctuates, with a fluctuating error of approximately $34 \mathrm{~mm} / \mathrm{s}$, but soon returns to its original steady-state value, as shown in Figure 8(b). From Figure 8(b) can be seen, the main, driven shaft in the start and external load interference action, a short period of time has a larger synchronization error value, but the transition process time is very short, quickly restored to the steady state value, indicating that the electric putter synchronous control system has a strong anti-jamming ability and robustness.

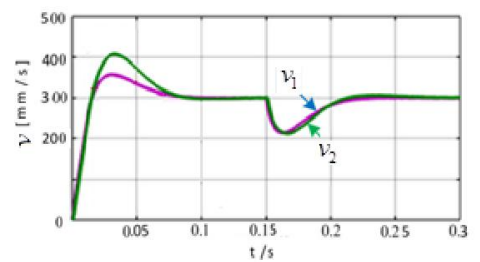

(a) Step response curve of the main, driven axis $\mathrm{Y}$ mechanism 


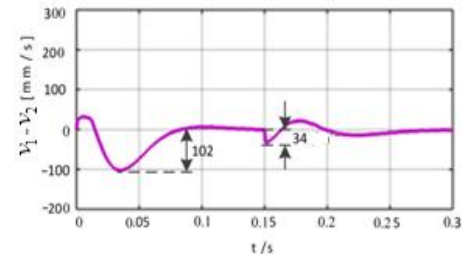

(b) Main, from axis speed synchronization error response curve

Fig. 8. Speed Response Characteristics Curve for Electric Pushrod

Synchronization System

\section{Conclusions}

(1) The synchronous control system of wind tunnel electric putter based on the deviation coupling mechanism of output displacement is proposed, and the structure and composition of the synchronous control system are introduced.

(2) For the wind tunnel model electric putter $\mathrm{Y}$ mechanism synchronous control system, the pushrod mechanical system, current control ring, speed control ring and position control ring, respectively, to establish a dynamic mathematical model;

(3) The three-ring synchronous control system of the electric putter Y mechanism of the wind tunnel model was adjusted, and the three-ring synchronous control system of the electric putter Y mechanism with excellent dynamic performance was designed.

(4) The performance simulation results of the electric putter Y mechanism synchronous control system of the wind tunnel model are analyzed, and the results show that the system has fast synchronous response speed, high synchronous control displacement and speed accuracy, and the system has strong robustness.

\section{Acknowledgment}

The authors gratefully acknowledge the reviewers for their comments and suggestions. This research was supported by the Natural Science Foundation of Jiangsu Province [grant number BK20190912], the Natural Science Foundation of China [grant number 61701430], the Project of 'Lyu Yang Jin Feng' Science and Technology Program.

\section{References}

(1) Niu H P, Peng Y H, He L, He M S, An approach to master speed control for coil box in hot strip mill. Metallurgical Industry Automation, 2011, 35(4): 8-14.
(2) Wu H, Zhang C F, Niu M G, Xiao Y Y, Leader-follower flocking for cooperative control of multiple motors. Changsha: IEEE Chinese Automation Congress, 2014: 47-52.

(3) Chen C S, Chen L Y. Robust Cross-Coupling Synchronous Control by Shaping Position Commands in Multiaxes System. IEEE Transactions on Industrial Electronics, 2012, 59(12): 4761-4773.

(4) Zhang Chenghui, Shi Qingsheng, Cheng Jin. A multi-motor synchronous control strategy based on adjacent coupling errors. Chinese electrical engineering transaction, 2007, 27(12): 59-63.

(5) Li L B, Sun L L, Zhang S Z, Yang Q Q, Speed tracking and synchronization of multiple motors using ring coupling control and adaptive sliding mode control. ISA Transactions, 2015, 58(1): 635-649.

(6) Chen Tianyi, Zhou Ping, et al., two-axis synchronous high-speed high-precision wind tunnel model mechanism control system design and commissioning. The 7th Three National Academic Exchange Paper of the Chinese Aerodynamics Association Measurement and Control Committee, Lujiang, Hunan Province. Chinese Aerodynamics Will Measurement and Control Professional Committee, 2018.8.

(7) Cao Yi. Research on dual-axis synchronous control technology. Wuhan: Huazhong University of Science and Technology, 2006. 